\title{
FORMAÇÃO INICIAL DOCENTE: SENTIDOS E SINGULARIDADES DA INICIAÇÃO CIENTÍFICA COMO POLÍTICA DE CIÊNCIA ${ }^{1}$
}

\author{
FORMACIÓN INICIAL DE MAESTROS: SIGNIFICADOS Y \\ SINGULARIDADES DE LA INICIACIÓN CIENTÍFICA COMO POLÍTICA \\ CIENTÍFICA
}

\author{
INITIAL TEACHER TRAINING: MEANINGS AND SINGULARITIES OF \\ SCIENTIFIC INITIATION AS A SCIENCE POLICY
}

\author{
Tereza Hortencia da Silva AZEVEDO ${ }^{2}$ \\ Marilene Batista da Cruz NASCIMENTO ${ }^{3}$ \\ Julita Batista da Cruz LOPES ${ }^{4}$
}

RESUMO: Esta pesquisa tem como objetivo compreender os sentidos e as singularidades (re)construídos pelos bolsistas/voluntários de iniciação científica (IC), com vistas à identificação das inter-relações com a formação inicial docente. Trata-se de um estudo teórico-empírico, de natureza qualitativa, que utilizou um questionário como instrumento para a coleta de dados trabalhados à luz da análise textual discursiva (ATD). As descobertas consentem concluir que as experiências vivenciadas na IC pelos participantes apresentam uma tendência atitudinal para a carreira acadêmica, também voltadas aos valores profissionais, respeitando-se o currículo e as práticas pedagógicas; aprendizagem para a autoria, interação e relação crítica; produção e difusão do conhecimento e pertinência social para a atuação nos espaços da profissão. Concluiu-se que a formação inicial de professores remete considerar as dificuldades existentes no processo de pesquisa dos acadêmicos e no trabalho do professororientador sobre a construção de saberes com o mundo, consigo e com o outro.

PALAVRAS-CHAVE: Formação inicial. Docente. Iniciação científica.

RESUMEN: Esta investigación tiene como objetivo comprender los sentidos y singularidades (re) construidas por los becarios / voluntarios de iniciación cientifica (CI), con el fin de identificar las interrelaciones con la formación inicial del profesorado. Se trata de un estudio teórico-empírico, de carácter cualitativo, que utilizó un cuestionario como instrumento para la recolección de datos trabajado a la luz del análisis textual discursivo (ATD). Los hallazgos permiten concluir que las experiencias vividas en CI por los participantes presentan una tendencia actitudinal hacia la carrera académica, también

\footnotetext{
${ }^{1}$ Esta pesquisa contempla discussão da tese da segunda autora com dados atualizados.

${ }^{2}$ Universidade Federal de Sergipe (UFS), São Cristóvão - SE - Brasil. Mestranda no Programa de Pósgraduação em Educação (PPGED/UFS). ORCID: https://orcid.org/0000-0001-8508-4239. E-mail: thaze@hotmail.com

${ }^{3}$ Universidade Federal de Sergipe (UFS), São Cristóvão - SE - Brasil. Docente no Departamento de Educação (DEDI) e do Programa de Pós-graduação em Educação. Doutorado em Educação (PUCRS). ORCID: http://orcid.org/0000-0002-6671-7907. E-mail: nascimentolene@yahoo.com

${ }^{4}$ Universidade Federal de Sergipe (UFS), São Cristóvão - SE - Brasil. Mestranda no Programa de Pósgraduação em Educação (PPGED/UFS). ORCID: https://orcid.org/0000-0002-7318-319X. E-mail: julita.lopes@yahoo.com.br
} 
enfocada en valores profesionales, respetando el currículo y las prácticas pedagógicas; aprendizaje para la autoría, interacción y relación crítica; producción y difusión de conocimiento y relevancia social para actuar en los espacios de la profesión. Se concluyó que la formación inicial de los docentes se refiere a considerar las dificultades existentes en el proceso de investigación de los académicos y en el trabajo del docente-asesor sobre la construcción del conocimiento con el mundo, contigo y con los demás.

PALABRAS CLAVE: Formación inicial. Profesor. Iniciación Cientifica.

ABSTRACT: This research aims to understand the meanings and singularities (re)constructed by scientific initiation (SI) scholarship holders/volunteers, with a view to identifying interrelations with initial teacher education. This is a theoretical-empirical study, of qualitative nature, which used a questionnaire as an instrument for the collection of data worked in the light of discursive textual analysis (ATD). The findings allow us to conclude that the experiences experienced in SI by the participants present an athedinal tendency towards the academic career, also focused on professional values, respecting the curriculum and pedagogical practices; learning for authorship, interaction and critical relationship; production and dissemination of knowledge and social pertinence for the performance in the spaces of the profession. It was concluded that the initial training of teachers refers to considering the difficulties existing in the research process of the students and in the work of the teacher-advisor on the construction of knowledge with the world, with himself and with theother.

KEYWORDS: Initial formation. Teacher. Scientific initiation.

\section{Introdução}

A iniciação científica (IC) como objeto de estudo decorreu do objetivo de entender os processos formativos das políticas públicas de ciência. O significado de política, aqui, não tem relação com os partidos políticos e eleições, o propósito é distinguir as abordagens de política derivada da ciência política, a qual mantém sua atenção à ação do Estado.

Souza (2006) trata da política pública sobre a perspectiva holística que situa o território de várias disciplinas, teorias e modelos analíticos. Assim, a política pública pertence, formalmente, ao ramo da ciência política, mas não se resume a ela, haja vista ser objeto analítico de diversos campos de conhecimento, a política pública (em geral) e a política social (em particular)

[...] são campos multidisciplinares, e seu foco está nas explicações sobre a natureza da política pública e seus processos. Por isso, uma teoria geral da política pública implica a busca de sintetizar teorias construídas no campo da sociologia, da ciência política e da economia. As políticas públicas repercutem na economia e nas sociedades, daí porque qualquer teoria da 
política pública precisa também explicar as inter-relações entre Estado, política, economia e sociedade (SOUZA, 2006, p. 25).

Esses aspectos permitem conceber a IC como uma política de ação pública que estimula a produção de conhecimentos em educação no Brasil, com relação à graduação, especificamente, na vertente da pesquisa. A IC foi criada em 1951, juntamente com o Conselho Nacional de Desenvolvimento Científico e Tecnológico ( $\mathrm{CNPq}$ ), órgão ligado diretamente ao Ministério da Ciência, Tecnologia e Inovação (MCTI). A criação do CNPq ocorreu na mesma época do início do financiamento da atividade de IC, mediante liberação de bolsas anuais de fomento à pesquisa na graduação. Entretanto, esse órgão não é o único para tal fim, há também as Fundações de Amparo à Pesquisa (FAP).

A estrutura funcional do CNPq compreende uma Diretoria Executiva responsável pela gestão da instituição e um Conselho Deliberativo, a qual atua na política institucional. O Regimento Interno do CNPq - Título I, Capítulo I, Art. $2^{\circ}$ - determina a esse órgão a missão de "[...] promover e fomentar o desenvolvimento científico e tecnológico do país e contribuir na formulação das políticas nacionais de ciência e tecnologia” (BRASIL, 2010, p. 1). Assim, a história do $\mathrm{CNPq}$ tem relação direta com o desenvolvimento e institucionalização da ciência e tecnologia no Brasil.

Nos dias atuais, tal órgão tem a missão de apoiar a pesquisa por meio de chamadas públicas e formar recursos humanos via concessão de bolsas, as quais se destinam a instituições, programas de pós-graduação (ou pesquisadores individualmente) para promover a formação e o aperfeiçoamento dos pesquisadores do país. As concessões dessas bolsas atendem aos programas de pós-graduação, a editais ou convênios com recursos próprios do CNPq ou de outras instituições públicas e privadas. As cotas podem ser concedidas a pesquisadores, cursos de pós-graduação e instituições de ensino, pesquisa e desenvolvimento tecnológico.

Os dados do CNPq, período de 2013-2018, revelam que já foram concedidas 611.984 mil bolsas de IC e de iniciação tecnológica (IT), assim distribuídas: Programa Institucional de Bolsas de Iniciação Científica (PIBIC) 466.554 (76,23\%); Programa de Iniciação Científica da Olimpíada Brasileira de Matemática das Escolas Públicas (PIC-OBMEP) 31.702 (5,18\%); Programa Institucional de Bolsas de Iniciação Científica para o Ensino Médio (PIBIC-EM) 27.717 (4,52\%); Programa Institucional de Bolsas de Iniciação em Desenvolvimento Tecnológico e Inovação (PIBITI) 75.919 (12,4\%); Programa Institucional de Iniciação Científica (PIBIC-Af) 7.480 (1,22\%); Programa de Iniciação Científica Júnior (ICJ) 2.622 (0,42\%) (PAINEL DE INVESTIMENTOS CNPq, 2019). 
As bolsas de IC atendem a qualquer área do conhecimento para estudantes de graduação que participarem de um projeto de pesquisa com um professor-orientador. Essa configuração associada à profissão docente permite adotar a concepção de um profissional reflexivo e da reflexão-na-ação como estratégia que fundamenta a epistemologia da prática, tendo repercussões, tanto do ponto de vista da pesquisa didática, quanto da formação de professores.

A Universidade Federal de Sergipe (UFS) oferta cursos de licenciatura e bacharelado em cinco campi, localizados nos municípios de Aracaju (Saúde), São Cristóvão, Itabaiana, Laranjeiras e Lagarto. São 106 cursos presenciais ofertados, sendo 72 bacharelados e 34 licenciaturas, as quais contemplam áreas de Ciências Biológicas e da Saúde, Ciências Exatas e da Terra, Ciências Humanas e Sociais Aplicadas, Linguística, Letras e Artes ${ }^{5}$.

Acredita-se que o docente é um cientista social cuja formação focada na pesquisa assegura a construção de conhecimentos e saberes que extrapolam os limites da disciplinaridade. Tal discussão perpassa pela formação pessoal e cultural e pelas perspectivas da formação inicial do professor que nos conduz às categorias de análise desta investigação. Tem-se, então, como objetivo compreender os sentidos e as singularidades (re)construídos pelos bolsistas/voluntários de IC, com vistas à identificação das inter-relações com a formação inicial de professores.

\section{Trilha metodológica da pesquisa}

O percurso teórico-metodológico deste estudo, quanto à natureza dos dados, baseou-se em uma abordagem qualitativa pela necessidade de se compreender a subjetividade, os sentidos e os significados da IC, como política de ciência, na graduação, especificamente, nos cursos de formação inicial.

O trabalho iniciou-se com conceitos e sínteses. Ao longo da caminhada visou-se (re)construir e compreender as categorias do estudo por meio da reflexão e da análise dos sentidos e das singularidades construídos no contexto da IC, bem como sua relação com a licenciatura. Os sujeitos desta investigação foram bolsistas/voluntários vinculados aos professores-orientadores de Programas de Iniciação Científica (PIC), do curso Pedagogia, Departamento de Educação/Campus Professor Alberto Carvalho/UFS, no período de 20172018. Buscou-se, junto ao lattes desses profissionais, localizar os bolsistas/voluntários participantes de projetos de IC no período indicado. Foram identificados 11 discentes, dos

\footnotetext{
${ }^{5}$ Disponível em: http://prograd.ufs.br/pagina/cursos-gradua-ofertados-7756.html. Acesso em: 19 set. 2020.
} 
quais oito responderam ao questionário on-line, compondo a população pesquisada (cinco bolsistas e três voluntários). Esse instrumento teve 16 questões abertas e fechadas, distribuídas em três etapas: dados de identificação, IC e formação de professores.

No tocante à faixa etária, os sujeitos desta pesquisa tinham idades variadas, a saber: uma bolsista e um voluntário entre 16 e 20 anos; duas bolsistas, duas voluntárias e um voluntário entre 21 e 25 anos; uma mulher bolsista entre 31 e 35 anos. Do total de participantes, dois estão no quarto período do curso de Física (1) e de Pedagogia (1); quatro estão no sexto período do curso de Pedagogia (2), de Física (1) e de Sistemas de Informação (1); no sétimo período de Letras (1) e no oitavo período de Pedagogia (1). Quanto à localidade de residência, três moram em Itabaiana, dois em Frei Paulo e um em cada uma das cidades a seguir: Aracaju, Campo do Brito e Nossa Senhora Aparecida.

Na conclusão da primeira etapa do questionário, registra-se a participação em grupos de pesquisa e a experiência em pesquisa como estímulos à produção científica. Dos oito sujeitos, apenas um voluntário indicou não pertencer a um grupo de pesquisa e dois afirmaram que já participaram de outra experiência de IC. Nesse sentido, "[há] uma convergência no meio acadêmico acerca dos grupos de pesquisa serem lócus de difusão e produção de ciência, caracterizando-se também como uma atividade orientada, dentro dos parâmetros da educação superior que desenvolve pesquisa” (NASCIMENTO, 2016, p. 199), em instituições de ensino superior (IES), sendo que a base do Diretório de Grupo de Pesquisa (DGP),

[...] disponibiliza informações sobre pesquisadores, estudantes e técnicos, bem como as linhas de pesquisa em andamento, as especialidades do conhecimento, aos setores de aplicação envolvidos, a produção científica e tecnológica. E de acordo com o censo bianual desse diretório, em 2014, os grupos de pesquisa com maior incidência são da área de Ciências Humanas atingindo um percentual de $21 \%$ (7.408) de um total de 35.424 grupos consolidados (NASCIMENTO, 2016, p. 199).

As áreas de Humanas, Sociais Aplicadas e Linguística, Letras e Artes, juntas, atingem $42 \%$ (14.703) do total de grupos de pesquisa no Brasil; Ciências Biológicas e da Saúde têm 26\% (9.259); Engenharias e Computação, Exatas e da Terra e Agrárias perfazem um total de $32 \%$ (11.462). Os dados revelam que a área de Humanidades têm mais grupos e recebem menos auxílio às pesquisas dos órgãos de fomento.

Após essas etapas de caracterização do perfil dos investigados, avançou-se para a apropriação dos resultados por meio da análise textual discursiva (ATD) que descreve esse tipo de estudo 
[...] como um processo auto-organizado de construção de compreensão em que novos entendimentos emergem de uma sequência recursiva de três componentes: desconstrução dos textos do corpus, a unitarização; estabelecimento de relações entre os elementos unitários, a categorização; o captar do novo emergente em que a nova compreensão é comunicada e validada (MORAES, 2003, p. 192).

A ATD aborda um ciclo de operações que visa à unitarização/desconstrução do material do corpus para se mover em direção à categorização das unidades de análise. Nessa fase, optou-se por caracterizar os sujeitos com letras e números, por exemplo: idade do pesquisado, H para homem e M para mulher; nome PIBIC ou PICVOL (20HPICVOL). Em seguida, emergiram compreensões e aprendizagens constituídas de auto-organização. A terceira etapa do ciclo congregou a explicitação das luzes sobre o fenômeno em estudo em forma de metatexto, potencializando a emergência de novos conhecimentos.

No tocante aos princípios éticos, mencionam-se, como base, as diretrizes divulgadas pelo $\mathrm{CNPq}$ publicadas para promover a ética na pesquisa científica. $\mathrm{O}$ documento descreve diretrizes que enfatizam, entre outros aspectos, a necessidade de dar créditos às fontes do trabalho, utilizando citações; reproduzir o significado exato das ideias ou fatos apresentados pelo autor original; apresentar os resultados de um estudo único complexo como um todo coesivo, no entanto, não é considerado ético que esses sejam fragmentados em manuscritos individuais.

Nessa perspectiva, esta pesquisa investigou as inter-relações dos bolsistas/voluntários de IC nos cursos de formação inicial a partir dos padrões éticos institucionalizados, com vistas à promoção da autoria responsável dentro do princípio da idoneidade por meio da aplicação do Termo de Consentimento Livre Esclarecido (TCLE). Diante disso, a ética supõe domínio dos princípios que regulamentam o aceitável na obtenção, no uso e na difusão do conhecimento de um campo do saber, e neste estudo, não se limitou ao fazer e comunicar, mas à utilidade das teorias e dos conceitos estudados.

\section{Sentidos e singularidades da iniciação científica na formação}

Esta etapa representa o desenvolvimento das categorias de análise encontradas na ATD, a saber: profissão/carreira acadêmica; escrita científica/opinião crítica e atividades acadêmicas/pesquisa/conhecimento; dedicação/compromisso. Cabe ressaltar que essas categorias emergiram da unitarização e categorização do corpus, constituídas pelos achados da pesquisa. 
A IC é um dispositivo que possibilita a (re)construção do conhecimento por meio da pesquisa nas IES. Por certo, o conceito dessa política nas universidades brasileiras foi formado como uma atividade que inicia o graduando no campo da ciência e permite a vivência de experiências vinculadas aos projetos de pesquisa, elaborados e desenvolvidos por um docente-orientador. Assim,

[...] a IC contribui para a formação sistemática e orientada da consciência do estudante acerca da importância e da acessibilidade ao processo de investigação, bem como da comunicação e utilização de seus resultados. Por meio desse dispositivo, os universitários são inseridos em atividades regulares de pesquisa, mediante planejamento e acompanhamento, visando construir conhecimentos na perspectiva de pesquisadores experientes (MASSI; QUEIROZ, 2010 apud NASCIMENTO, 2016, p. 77).

Quando os bolsistas foram indagados sobre como surgiu o interesse pela IC, as respostas foram diversificadas a respeito do desenvolvimento de habilidades voltadas à escrita, à carreira acadêmica, à produção do Trabalho de Conclusão de Curso (TCC), à construção de conhecimento, conforme relatos:

Por busca questionar e analisar um tema. (20HPICVOL).

As pessoas me incentivaram para melhorar a escrita e o desenvolvimento do futuro TCC. (21MPIBIC).

Depois de uma determinada disciplina, surgiu a curiosidade de entender como é o processo de educação no Brasil e também, depois do contato com uma das primeiras escolas do Estado, procurei entender através da pesquisa, determinado tema. (22MPIBIC).

A fim de melhorar a minha escrita e porque tenho interesse em seguir carreira acadêmica. (22MaPICVOL).

Pretendo seguir carreira acadêmica e amadurecer enquanto pesquisador. (23HPIBIC).

Desejo seguir uma carreira acadêmica. (33MPIBIC).

A IC como espaço de formação emerge como possibilidade de se apreender conteúdos e (re)significar conhecimento. Afirma-se, assim, que formar-se na IC é transcender o ensino baseado na memorização ou reprodução. Trata-se de se ter uma “[...] possibilidade de criar espaços de participação, reflexão e formação para que as pessoas aprendam e se adaptem para poder conviver com a mudança e a incerteza" (IMBERNÓN, 2011, p. 15).

Para Charlot (2014, p. 50), “[só] pode aprender quem desenvolve uma atividade intelectual para isso e, portanto, ninguém pode aprender em vez do outro. [...] por mais semelhantes que sejam os seres humanos, são também singulares e, logo, diferentes". Isso significa que a aprendizagem tem relação com o sentido e o significado do objeto estudado. 
Os sentidos e singularidades aparecem quando os bolsistas/voluntários indicaram as atividades de IC das quais mais gostavam. Os resultados podem ter relação com as ações realizadas em seus respectivos cursos, por exemplo: os bolsistas/voluntários que cursam Pedagogia elegem fichar, analisar, debater e questionar o tema em estudo; participantes que cursam Física preferem apresentar; de Geografia, destacam o questionamento e os de Sistemas de Informação sinalizam o ato de investigar em si, de acordo com as narrativas:

\section{Descubro novas coisas, cresço academicamente. (19MPIBIC). Aprendi mais a fazer trabalhos acadêmicos. (21MPIBIC). \\ Consegui desenvolver maior entendimento sobre pesquisa e interesse em dar continuidade ao processo de formação acadêmica (mestrado, doutorado). (22MPIBIC). \\ Tenho oportunidade de aprender a escrita cientifica e de estudar com um maior foco sobre o tema que tenho interesse em pesquisar. (22MaPICVOL). Posso formar opiniões mais críticas e tirar melhores conclusões daquilo em que estou trabalhando. (22MbPICVOL). \\ Consigo ampliar minhas leituras, produção textual, discutir sobre diversas pesquisas que estão sendo desenvolvidas. (23HPIBIC).}

Percebe-se que os incluídos na IC, que conseguiram vivenciar essa experiência de caráter científico, ratificam as contribuições à melhoria da sua formação no curso $^{6}$, ao do desenvolvimento pessoal por meio dos elementos e da função social da ciência, da ressignificação de saberes e apropriação da linguagem científica, com vistas à potencialização da práxis docente.

Pesquisou-se, ainda, sobre as atividades das quais o bolsista/voluntário menos gostava nos projetos de IC. O resultado sinalizou a criação de tabelas (parte quantitativa), o tempo corrido para as atividades, o apoio de terceiros, o momento da socialização, prazos e cobranças, relacionar os autores estudados com a pesquisa, muita leitura, dificuldade em localizar material. Dentro dessas dificuldades, cabe evidenciar que uma IC de qualidade contempla atividades de escrita científica, aprendizado e conhecimento, com vistas ao desenvolvimento de diferentes habilidades que envolvem disponibilidade de tempo, comprometimento e disciplina. Essas questões foram apresentadas pelos pesquisados, a saber:

Onde existem as correções de erros, [...] aprende-se corretamente. (19MPIBIC).

[Faz-me] refletir sobre o tema. (20HPICVOL).

Impulsiona o estudante para novas buscas. (22MPIBIC).

Aprende a escrever melhor e que publica algum artigo. (22MaPICVOL).

${ }^{6}$ Defende-se, neste estudo, que a formação de professores "[...] deve privilegiar a realidade social [...], contextualizar [...] de modo significativo, construir elementos que auxiliem e orientem o saber fazer dos estudantes para resolver problemas cotidianos [...], visando ao estímulo da criatividade [...], ao trabalho coletivo e cooperativo, de maneira ética e ligado à cidadania" (FISTAROL; FISCHER; WENDERLICH, 2019, p. 348). 
Levanta discussões, debates, ou seja, troca informações e experiências. (22MbPICVOL).

Estimula a produção e progresso acadêmica do aluno. (23HPIBIC).

Capacita e incentiva o aluno a pesquisar. (33MPIBIC).

Me faz refletir sobre o tema (20HPICVOL)

Estimula a produção e progresso acadêmica do aluno (23HPIBIC)

Essa perspectiva relaciona-se com a ideia de que toda formação superior pressupõe uma experiência de IC para o desenvolvimento do espírito científico, sendo a pesquisa na universidade um pilar indissociável do ensino e da extensão no campo da formação inicial da profissão docente, com vistas a produzir ciência, inclusive, a pedagógica. Para Martins, Antunes e Monteiro (2019), a formação deve atender as exigências da contemporaneidade, ultrapassando os muros das universidades para atender ao cotidiano vivido e praticado nos espaços de aprendizagens formais, com compromisso e comprometimento social.

Assim, a experiência de ser bolsista/voluntário do PIBIC/UFS contribui para a futura profissão, quando direcionada à profissionalização. Por outro lado, destaca-se a probabilidade de se seguir a carreia acadêmica pela maioria dos participantes, sendo necessárias aprendizagens voltadas à escrita científica, senso crítico e formação profissional. Assim, a IC

[...] despertou coisas que eu não sabia que existia, por exemplo, a própria pesquisa, a leitura, a escrita. Abriu [minha] visão e [...] acredito crescer [acadêmica] e profissionalmente com a experiência atual e futura. (19MPIBIC).

[pode] melhorar meu desenvolvimento com as atividades acadêmicas e explorar mais conteúdos. (21MPIBIC).

[proporciona] uma abertura e um passo no mundo do conhecimento cientifico e acadêmico, posteriormente, profissional. (22MPIBIC).

[estimulou] o interesse em fazer mestrado e, possivelmente, doutorado e tenho certeza de que a experiência no PIBIC irá me ajudar muito. Se soubesse o quanto o PIBIC amplia os conhecimentos já teria entrado [...]. (22MaPICVOL)

[...] me ajuda a contextualizar a minha futura profissão, me faz ver o que a envolve, o que tem de melhor e de pior na sua prática. (22MbPICVOL).

[aprendo] sobre diversos contextos, sujeitos que poderei encontrar em minha jornada enquanto acadêmico/professor. (23HPIBIC).

Nota-se o reconhecimento do valor da IC para favorecer o desenvolvimento de habilidades diferenciadas no cotidiano da educação superior dos acadêmicos emerge categoricamente, bem como estimula a capacidade crítica diante dos problemas sociais, culturais e econômicos, com a elaboração de argumentos, via escrita e oralidade. Nessa perspectiva, a IC nos cursos de formação inicial constitui-se como fundamental para a trajetória profissional, configurando-se como um espaço reflexivo sobre a construção e o fortalecimento da apropriação de aprendizagens de conteúdos científicos diferenciados e da 
vivência de experiências colaborativas para a profissão. Essa condição remete considerar as dificuldades existentes no processo de pesquisa, bem como no trabalho do professororientador sobre a construção de saberes com o mundo, consigo e com o outro. De acordo com Bersch e Schlemmer (2018), a formação, ainda, pode contribuir para o desenvolvimento de habilidades com potencial criativo e inventivo, visando à construção de práticas reticulares que promovam mudança nas culturas da universidade.

Por fim, destaca-se que a formação inicial de professores perpassa por questionamentos e vulnerabilidades que a separa da produção do saber científico, sendo de relevância a ampliação da oferta de IC para as transformações no campo institucional e no desenvolvimento profissional atrelado à uma formação em pesquisa ${ }^{7}$ que se constrói no cotidiano.

\section{Algumas considerações}

A configuração da IC apresenta-se como uma política pública que promove acessibilidade aos avanços nas redes da pesquisa, sendo possível a implementação de mecanismos capazes de estimular a mobilização da comunidade científico-tecnológica. Além disso, a IC contribui para a redução das diferenças socioeconômicas entre regiões e países com vistas a favorecer a pesquisa em tempos de internacionalização na educação superior.

As descobertas, neste estudo, consentem concluir que as experiências dos bolsistas/voluntários vivenciadas na IC apresentam uma tendência atitudinal para a carreira acadêmica, também voltadas aos valores profissionais, respeitando-se o currículo e as práticas pedagógicas de pesquisa; aprendizagem para a autoria, interação e relação crítica; produção e difusão do conhecimento com qualidade e pertinência social para a atuação profissional.

Por outro lado, a insuficiente quantidade de bolsas caracteriza a IC como uma política seletiva e excludente, criando prerrogativas para alguns e exclusão para outros. Portanto, o caráter pragmático desse dispositivo nas universidades, pública e privada, como indutor da pós-graduação, não pode perder de vista sua especificidade na graduação, que, de fato, forma e certifica o cientista social, entendida toda a ciência como humana, portanto, social.

7 Defende-se que a formação em pesquisa seja fomentada na educação básica e superior, visando ao desenvolvimento pessoal e profissional por meio das experiências, da reflexão crítica, do empoderamento para elaboração e execução de ações com vistas à transformação social (ALVES; FIALHO; LIMA, 2018). 
AGRADECIMENTOS: Esta pesquisa recebeu financiamento da Coordenação de Aperfeiçoamento de Pessoal de Nível Superior (Capes) pelo Programa de Pós-graduação em Educação (PPGED/PROAP/UFS) e fez parte do projeto "Formação Inicial de Professor e a Relação com o Saber: um estudo com egressos de iniciação científica", via PIBIC/UFS/CNPq, com o apoio da bolsista Arlene de Almeida Pereira Siqueira.

\section{REFERÊNCIAS}

ALVES, F. C.; FIALHO, L. M. F.; LIMA, M. S. Formação em pesquisa para professores da educação básica, Revista Tempos e Espaços em Educação, São Cristóvão, v. 11, n. 27, p. 285-300, out./dez. 2018.

BERSCH, M. E.; SCHLEMMER, E. Formação continuada em contexto híbrido e multimodal: ressignificando práticas pedagógicas por meio de projetos de aprendizagem gamificados.

Revista Tempos e Espaços em Educação, São Cristóvão, v. 11, n. 1, p. 71-92, 2018.

BRASIL. A iniciação científica: uma estratégia eficaz de transformação. Conselho Nacional de Desenvolvimento Científico e Tecnológico (CNPq). Brasília, DF: CNPq, 2010.

CHARLOT, B. Da relação com o saber às práticas educativas. São Paulo: Cortez, 2014.

CNPq. Conselho Nacional de Desenvolvimento Científico e Tecnológico. 2019. Disponível em: http://cnpq.br/apresentacao-programas/. Acesso em: 15 dez. 2019.

FISTAROL, C. F. S.; FISCHER, A.; WENDERLICH, R. C. C. A base nacional comum curricular e a formação de professores de Língua Inglesa: desafios e possibilidades. Revista on-line de Política e Gestão Educacional, Araraquara, v. 23, n. 2, p. 341-355, maio/ago. 2019.

IMBERNÓN, F. Formação docente e profissional: formar-se para mudança e a incerteza. 9. ed. São Paulo: Cortez, 2011.

MARTINS, E. B. A.; ANTUNES, K. C. V.; MONTEIRO, S. S. Formação continuada de professore e educação inclusiva: os saberes fazeres docentes em diálogo com a extensão universitária. Revista on-line de Política e Gestão Educacional, Araraquara, v. 23, n. 2, p. 877-896, maio/ago. 2019.

MORAES, R. Uma tempestade de luz: a compreensão possibilitada pela análise textual discursiva. Ciência \& Educação, São Paulo, v. 9, n. 2, p. 191-211, 2003.

NASCIMENTO, M. B. C. Iniciação científica em redes colaborativas e formação universitária de qualidade: a perspectiva do egresso (2007-2013). 2016. 282 f. Tese (Doutorado em Educação) - Pontifícia Universidade Católica do Rio Grande do Sul, Porto Alegre, 2016. 
PAINEL DE INVESTIMENTOS CNPq. Programas Institucionais de Iniciação Científica e Tecnológica do CNPq. Disponível em: http://memoria.cnpq.br/painel-de-investimentos. Acesso em: 10 dez. 2019.

PIBIC. Programa Institucional de Bolsas de Iniciação Científica. Disponível em: http://cnpq. br/pibic. Acesso em: 10 out. 2019.

PIBIC-Af. Programa Institucional de Iniciação Científica nas Ações Afirmativas. Disponível em: http://cnpq.br/pibic-nas-acoes-afirmativas. Acesso em: 10 out. 2019.

PIBIC-EM. Programa Institucional de Bolsas de Iniciação Científica para o Ensino Médio. Disponível em: http://cnpq.br/pibic-ensino-medio. Acesso em: 10 out. 2019.

PIBITI. Programa Institucional de Bolsas de Iniciação em Desenvolvimento Tecnológico e Inovação. Disponível em: http://cnpq.br/pibiti. Acesso em: 10 out. 2019.

PIC-OBMEP. Programa de Iniciação Científica da Olimpíada Brasileira de Matemática das Escolas Públicas. Disponível em: http://cnpq.br/pic-obmep. Acesso em: 10 out. 2019.

PROGRAD. Pró-reitoria de Graduação da Universidade Federal de Sergipe. Disponível em: http://prograd.ufs.br/pagina/cursos-gradua-ofertados-7756.html. Acesso em: 10 set. 2019.

SOUZA, C. Políticas públicas: uma revisão da literatura. Sociologias, Porto Alegre, ano 8, n. 16, p. 20-45, jul./dez. 2006.

VISEU, A. S. A. S. C. Políticas públicas de ciência e a regulação da investigação educacional: estrutura e redes de investigadores. 2012. Tese (Doutorado em Educação) Universidade de Lisboa, Portugal, 2012.

\section{Como referenciar este artigo}

AZEVEDO, T. H. S.; NASCIMENTO, M. B. C.; LOPES, J. B. C. Formação Inicial Docente: sentidos e singularidades da iniciação científica como política de ciência. Revista on line de Política e Gestão Educacional, Araraquara, v. 24, n. 3, p. 1408-1419, set./dez. 2020. eISSN:1519-9029. DOI: https://doi.org/10.22633/rpge.v24i3.14262

Submetido em: 20/06/2020

Revisões requeridas: $15 / 08 / 2020$

Aprovado em: 30/08/2020

Publicado em: 01/09/2020 